\title{
Epileptic Seizure Detection in EEG Signal using EMD and Entropy
}

Inung Wijayanto, Achmad Rizal

School of Electrical Engineering

Telkom University

Bandung

Indonesia

iwijayanto@telkomuniversity.ac.id, achmadrizal@telkomuniversity.ac.id

\begin{abstract}
Epilepsy is a disease caused by abnormal electrical activity in the brain. One of the techniques for diagnosing epilepsy is by analyzing electroencephalogram (EEG) signals. Various techniques were developed by researchers to analyze epileptic seizure on EEG signals. Because of the nonlinear, non-Gaussian, and nonstationary nature of EEG signals, methods such as empirical mode decomposition (EMD) are often used for analysis on EEG signals. The intrinsic mode function (IMF) of the EMD is believed to provide different information for normal EEG and seizure signals. Some features are taken from the $I M F$ such as statistical features and spectral features. One of the differences between normal signals and abnormal signals is signal complexity where one of the metrics for measuring them is entropy. Several research used entropy combined with first and second order statistical features. In this study, only one entropy feature used to characterize each IMF produced from EMD for the classification of epileptic seizure EEG. Entropie used were Shannon entropy (ShEN), spectral entropy (SE), Renyi entropy (RE), and permutation entropy (PE). The highest accuracy produced by RE in eight IMF uses quadratic support vector machine (SVM) as the classifier. The accuracy of $97.3 \%$ with sensitivity of $97 \%$ and specificity of $99.75 \%$ was achieved for classification in three data classes. The developed method is able to produce high accuracy with a relatively small number of features.
\end{abstract}

Keywords: Electroencephalogram, Empirical Mode Decomposition, Entropy, Epilepsy, Classification

Received: 10 October 2018, Revised 12 January 2019, Accepted 29 January 2019

DOI: $10.6025 / \mathrm{jes} / 2019 / 9 / 2 / 44-54$

(C) 2019 DLINE. All Rights Reserved

\section{Introduction}

Epilepsy is a severe neurological problem faced by humanity [1][2]. For decades, humanity faced by the increasing number of people with epilepsy. Epilepsy case found at $0.8 \%$ of the world population, which is more than 50 million people [3]. Furthermore, it is mentioned by the WHO that the number of people with epilepsy was mostly found high in the third world and developing countries [4]. This significant number happened because of the lack of information and awareness about how epilepsy and how dangerous it for a human being [2], [4]. Indonesia, as one of developing country, faced the same problems, even worse, the people with epilepsy with their family often get a negative stigma. This condition eventually made a new social problem for them [5].

The conceptual definition of epilepsy defined by The International Leauge Against Epilepsy (ILAE) is "a disorder characterized by an enduring predisposition to generate epileptic seizure and by neurobiological, cognitive, psychological and social conse- 
quences of this condition. The definition requires the occurrence of at least one epileptic seizure" [6]. In other hands, operational definitions of epilepsy defined by Thurman et al., which adopted from the ILAE in 1993 [7], is "two or more unprovoked seizure occurring at least 24 hours apart" [8].

A seizure may cause an uncontrolled body movement of the patients. This condition may spread from one body part to the other body parts. Moreover, this condition may lead to a faint [2]. There is hope for the patient to cure the seizure symptoms; the first one is by consuming a regular medication. The success rate for this method is $66 \%$. The patient needs to undergo surgery if the medication failed to work. Unfortunately, surgery only gave another $8 \%$ success rate for curing the seizure. The rest is about $25 \%$ of people with epilepsy has no option left, they need to live with epilepsy in their entire life [9]-[11]. Due to the natural characteristic of seizure which cannot be predicted [9], the people with epilepsy have to face their most significant handicap and their primary source of worry [12].

Electroencephalography (EEG) signal, an electrical activity recording of the brain, has become another inexpensive option for the neurologist to determine the brainwave manifestation for the patient with epilepsy, sleep disorder, Alzheimer, and other mental illness [13]. A certified physician does EEG reading, interpretation, and reporting. Unfortunately to get a certified physician is another problem for health care facilities need to face. It considered unpractical for them to have a certified physician to standby 24/7 for long-term EEG monitoring and recording. EEG signal interpretation process has a subjective nature. There was a high chance of misinterpretation due to human error which leads to misdiagnosis [14].

Many computational models have developed to solve this problem, especially for seizure monitoring, and detection. Seizure detection techniques development can be done by using several processing domains such as time, frequency, wavelet, and EMD domain. A comprehensive review about the development in epileptic seizure detection can be found in [10] and [15]. We use the EMD domain as our approach to detect seizure in the EEG signal. EMD domain basically based on time-frequency analysis. The approach was independent from wavelet and Fourier domain which have a prior fixed basis. By nature, EMD was adaptive and did not need a prior fixed basis to analyze non-stationer and nonlinear signals [15], [16].

Previous EMD based research for classifying seizure in EEG signal shows promising developments. Shaikh et al. [17] used EMD to do seizure detection using ANN as the classifier. Tripathi et al. [18] used Fuzzy entropy and EMD to detect epileptic EEG signal. On the other hand, EMD and SVM were used to extract and recognize ictal EEG by Li et al. in [19].

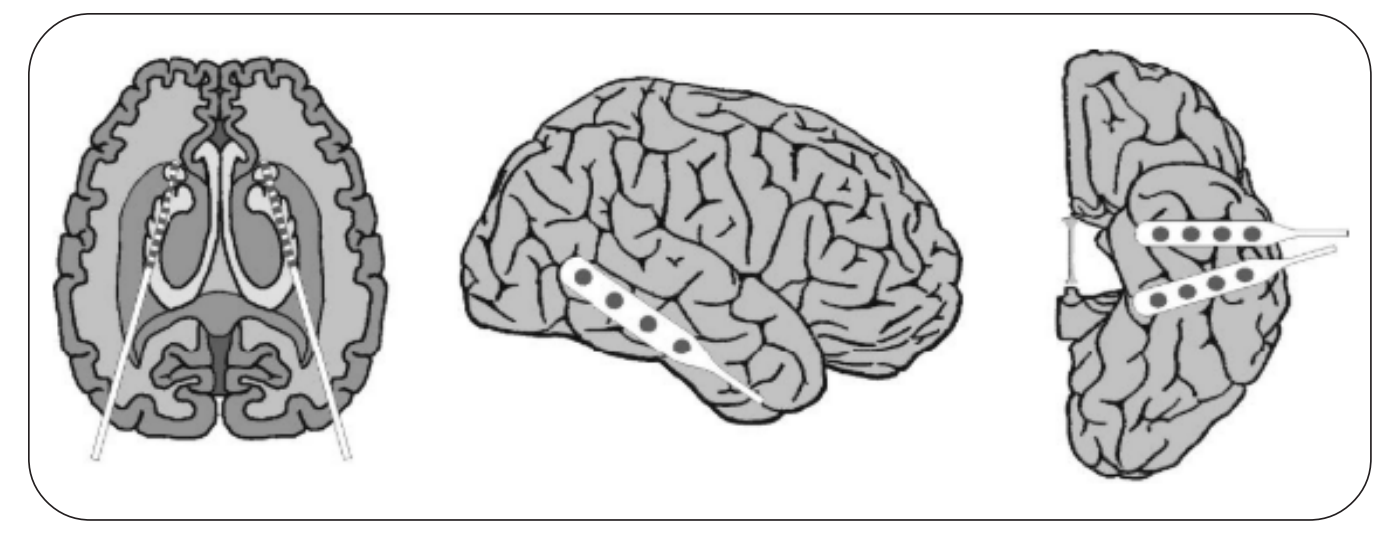

Figure 1. Electrodes location in Bonn University iEEG recording process

\section{Material and Method}

\subsection{Dataset}

Our research used EEG data from the University of Bonn. The data consist from two types of EEG recording, the first one is scalp EEG (sEEG) recording, and the second is intracranial EEG (iEEG) recording. The sEEG data recorded from healthy participants with eyes open, and closed condition named Set A, and Set B respectively. iEEG data recorded from people with epilepsy by placing depth electrodes symmetrically in the hippocampal formation. Strip electrodes placed in the lateral and basal regions of the neocortex. The segment of the dataset Set C and Set D, called interictal condition, were recorded from the depth electrodes. While the seizure condition, named Set E, were recorded from all depicted electrodes. Details of the location for each electrode can be 
seen in Fig. 1. Bonn University dataset use 128 channel amplifier system, and average common reference (AR) to remove pathological activity and eye movement in the signal. The data use $17.61 \mathrm{~Hz}$ as the sampling rate [20]. The classes of Bonn University dataset can be seen in Table 1 .

\subsection{Empirical Mode Decomposition}

Empirical Mode Decomposition is a non-stationer signal analysis technique proposed by Huang et al. [16]. EMD decomposed a signal into several intrinsic mode functions (IMF) and residue by removing local oscillation of the signal [21]. EMD became the initial part of Hilbert-Huang transform (HHT) to obtained instantaneous frequency (IF) of a signal [16]. The use of EMD can be found for lung sound analysis in several cases, such as noise identification, velcro or crackle identification [22]-[24]. EMD can be used for EEG signal, several research has been done to do ictal EEG classification [19], [25], seizure classification [26], [27], artifact removal [28]-[30], and brain death analysis [31].

\begin{tabular}{|l|l|l|}
\hline Set & Recording type & EEG Signal \\
\hline A & sEEG & Normal \\
B & sEEG & Normal \\
C & iEEG & Interictal \\
D & iEEG & Interictal \\
E & iEEG & Ictal \\
\hline
\end{tabular}

Table 1. Bonn University Dataset Classes

Given a signal $x(t)$, the EMD can be calculated using the following steps.

1. Identify the extrema of the signal, mean of upper and lower envelope of the signal $x(t)$, then check the condition for IMF.

2. Calculate the local mean of upper and lower-envelop using equation 1.

$$
m(t)=\frac{e_{\min }(t)+e_{\max }(t)}{2}
$$

3. Calculate the detail using $d(t)=x(t)-m(t)$

4. If the detail is not suitable with IMF's criteria, the process is repeated from step 1, $m(t)$ used as the new input.

5. If the detail is suitable with IMF's criteria, then the detail is choosen as tihe IMF $\left(k_{i}(t)=d(t)\right.$, where $i$ indicates the $i$-th IMF.

6. The choosen IMF removed from the signal using $n(t)=x(t)-k_{i}(t)$

7. Repeat the process from step $1, n(t)$ used as the new input.

$$
x(t)=\sum_{n} \mathrm{IMF}_{n}+\text { residue }
$$

Equation 2 generally describes those procedures, where can be decomposed into IMFs and its residue. Fig. 2 shows an example of the output of the EMD process in one signal from Set D which is epileptic no seizure EEG signal of Bonn University Dataset. It produced 11 IMFs and one residue. It is clear that the signal oscillations is slowing down as the IMF level increasing. Shahbakhti et al. use three IMFs to remove eyeblink from EEG signal [29], [30]. Eight IMFs are used by Yadav et al. to classify normal and apnea sleep based on EEG signal [32]. Other research using Bonn University dataset, such as conducted by Shaikh et al. [17], and Li et al. in [19] used five IMFs, while Martis et al. in [33], and Tripathi et al. in [18], used eight IMFs for the same dataset. In this research, we use 8 IMFs as the input for the feature extraction process. 


\subsection{Feature Extraction}

Feature extraction used in this research was based on entropy calculation. Entropy can be explained as one method to calculate the complexity of the signal. A signal with high entropy value indicates that the signal had a higher complexity compared to the one with lower entropy value. We explore the use of four types of common entropy to find out which one was suitable for the system.

\subsubsection{Shannon Entropy}

Shannon entropy (ShEN) represent the signal complexity [34]. For a signal $x(t)$ with $p_{i}$ as the sample value appearance probability, ShEN is obtained using equation 3 .

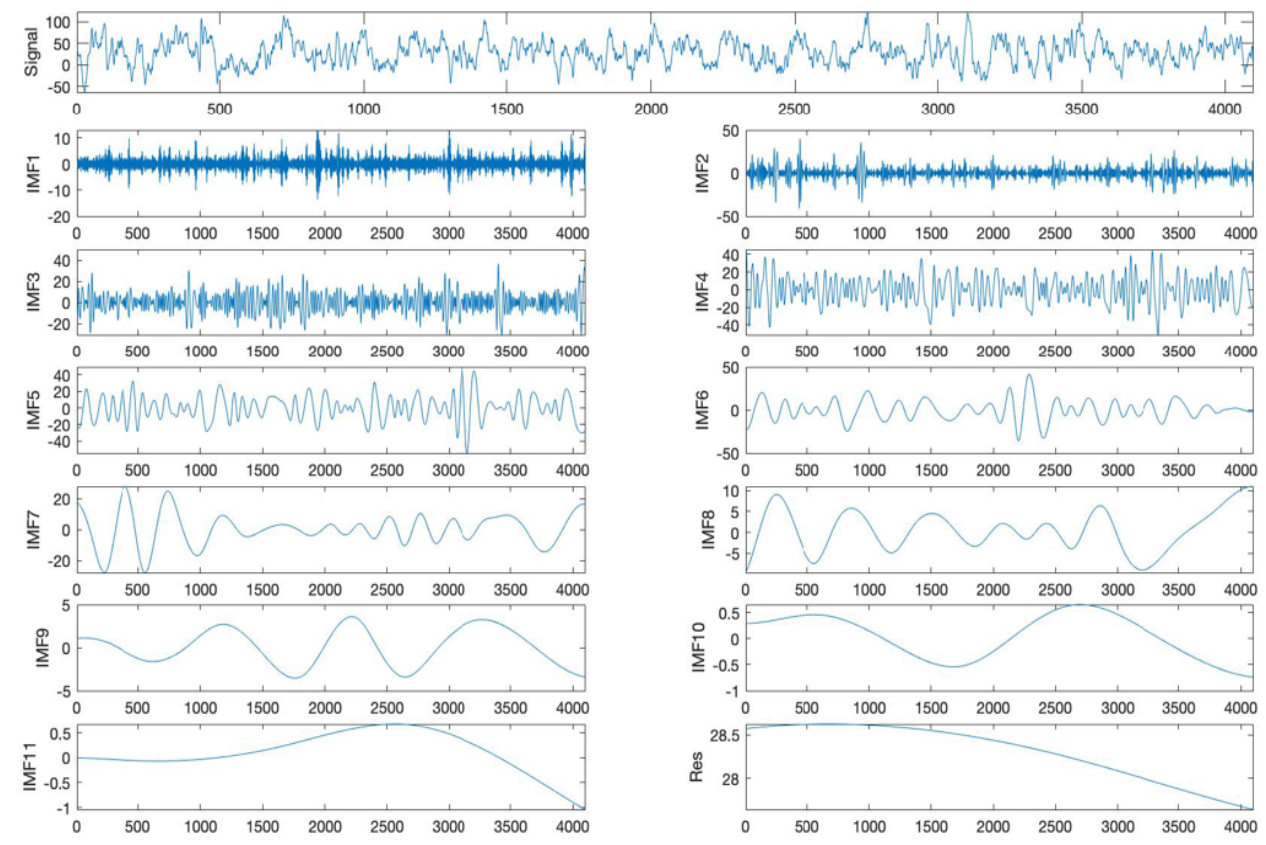

Figure 2. Sample of EMD result on Set D, Epileptic no seizure EEG signal, of Bonn University Dataset

$$
\operatorname{ShEN}=-\sum_{i=1}^{N} p_{i} \log _{2} p_{i}
$$

\subsubsection{Spectral Entropy}

The normalization of ShEN was called as spectral entropy (SEN). SEN was calculated from the signal spectrum which defines the irregularity of the histogram of the signal. The calculation can be done using equation 4 .

$$
\operatorname{SEN}=-\sum_{f=0}^{f h} p_{f} \log _{2}\left(\frac{1}{p_{f}}\right)
$$

$p_{f}$ indicates the power density of the frequency band, where the frequency limited from $f$ to $f_{h}$. Renyi Entropy Renyi entropy (RE) were the common form of entropy calculation [35]. Practically, entropy calculation was being called as RE for $q$ the in equation 5 equal with two.

$$
S_{q}=\frac{1}{1-q} \log _{2}\left(\sum_{i=1}^{N} p_{i}^{q}\right)
$$

\subsubsection{Permutation Entropy}

Permutation entropy (PE) calculate the signal complexity by identifying the code between group of signals [36]. PE find the permutation pattern from different elements of a signal. The calculation of PE can be done using equation 6 . 


$$
P E=-\sum_{j=1}^{n} p_{j} \log _{2} p_{j}
$$

Where $p_{j}$ indicates the relative frequency from a group of a possible pattern. $n$ indicate the permutation order with $n \geq 2$.

Eight IMFs from EMD were each processed using four types of entropy producing one feature for each IMF. These features then fed to the classifier to find out which has the best accuracy.

\subsection{Support Vector Machine (SVM)}

Support Vector Machine (SVM) is developed by Vapnik [37] in 1995. The specialty of this method is the generalization capability by only using a few parameters [38]. The SVM generalization can optimize and make the data more dependence. The performance of SVM is proved better and able to compete with another machine learning methods such as random forests, and artificial neural networks. The algorithm developed by Vapnik is focusing on how to maximizing the minimum separating hyperplane. In another word, this algorithm is to detect the shortest distance between the data's decision function [39].

We use linear, and non-linear (quadratic and cubic) SVM kernel. We use 5-fold cross-validation to determine the training and testing dataset. The dataset is first split into five datasets then, the four datasets are used as testing data. This process is repeated until all the split datasets are once used as the test data. The performance of this process is calculated by using sensitivity, specificity, and accuracy.

\subsection{Performance Evaluation}

Our work used SVM as the classifier. The suggested method performances were determined by using 5-fold cross-validation. This method randomly divides the data into five different mutually exclusive folds at the same sizes. Four-fold used as the training set, while one fold used as the testing set. This process was repeated 5 times. The performance of the classification method was measured by calculating sensitivity, specificity, and accuracy. Those three measurements expressed by the number of true positives (TP), true negative (TN), false positive (FP), and false negative (FN).

$$
\begin{aligned}
& \text { Sensitivity }=\frac{T P}{T N+F N} \times 100 \\
& \text { Sensitivity }=\frac{T N}{T N+F P} \times 100 \\
& \text { Accuracy }=\frac{T P+T N}{T P+F N+T N+F P} \times 100
\end{aligned}
$$

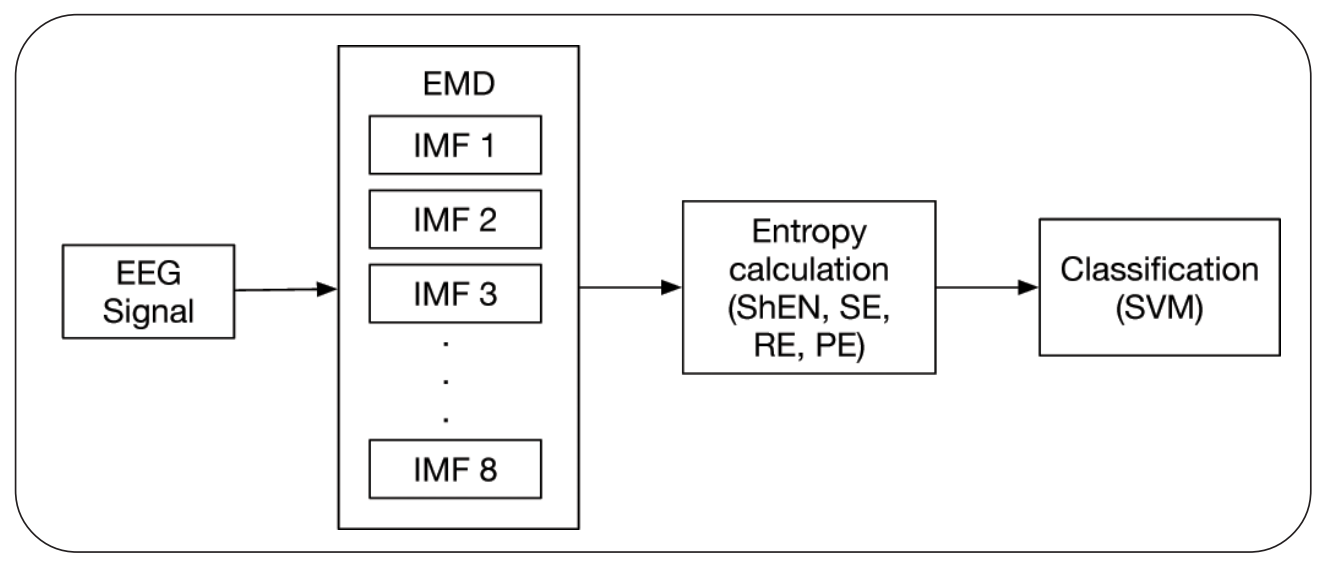

Figure 3. Seizure classification process 


\section{Result and Discussion}

We used the EEG signal from Bonn University dataset as the input of the system. It has five classes, normal healthy with open and closed eyes (Set A and B), epileptic no seizure or can be called as interictal condition (Set C and D), and seizure condition (Set E). Four types of entropy were used to calculate the first eight IMFs. After the feature calculation, the next step was feeding the features to the SVM to be classified. Brief of the seizure classification process can be seen in Figure 3.

Figure 4 shows the sample of eight first IMF from set E of Bonn University EEG dataset. Example of entropy calculation for the sample can be seen in Table 2 . After the calculation, every signal had eight features for each entropy.

Five-fold cross-validation technique was used to stabilize the performance of the classification process [40]. There was three scenario to do the classification. In the first scenario, we classified the data into three classes, normal healthy (Set A, and B), interictal (Set C, and D), and seizure (Set E). The second scenario, we reduce the number of normal and interictal set data. The classes then became normal healthy (Set B), interictal (Set C), and seizure (Set E). The third scenario, we classified all the dataset into five classes (Set A-E).

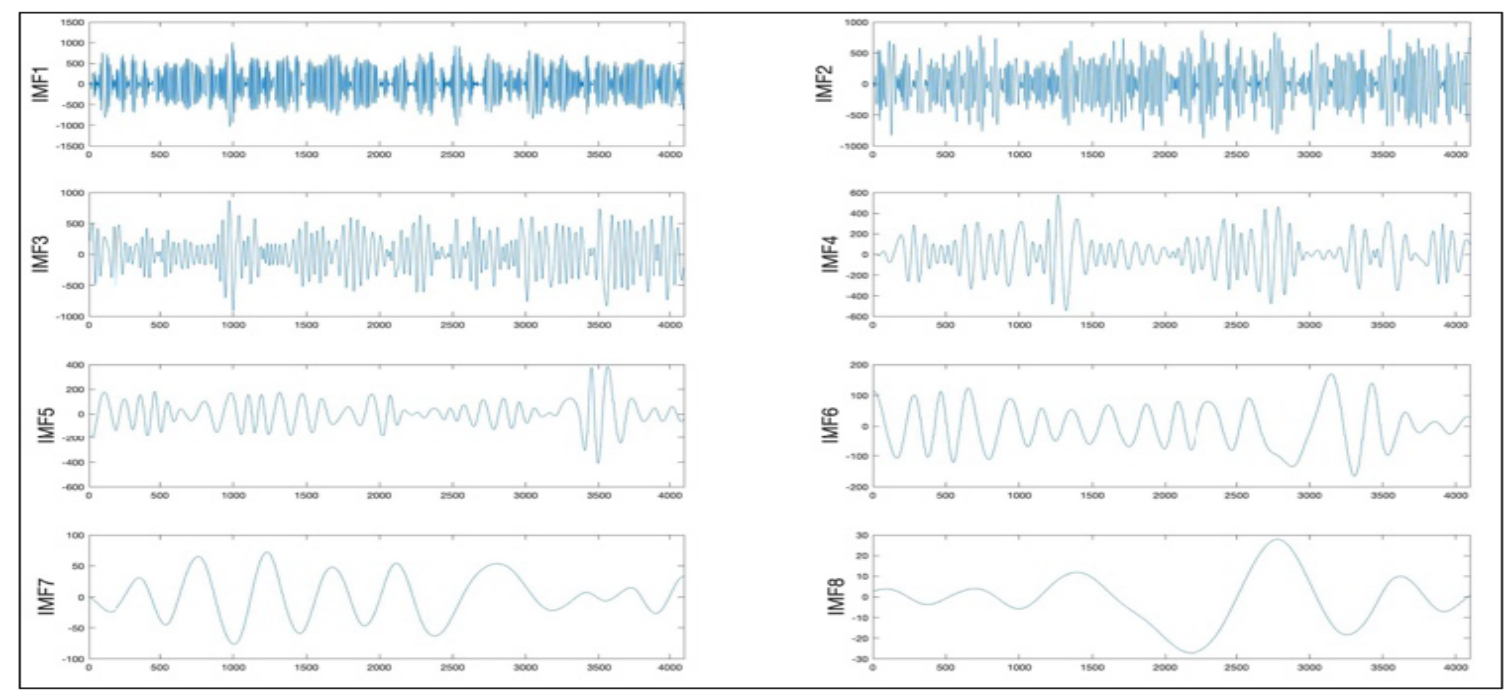

Figure 4. Eight first IMFs from Set E (Seizure) of Bonn University EEG dataset

\begin{tabular}{|c|c|c|c|c|}
\hline & & & & \\
\hline IMF1 & 1.0273097 & -19.974277 & 0.7954044 & 0.4860477 \\
IMF2 & 1.021009 & -19.800302 & 0.733289 & 0.373658 \\
IMF3 & 1.0143885 & -19.701716 & 0.6486532 & 0.2488318 \\
IMF4 & 1.0476189 & -18.595422 & 0.5677114 & 0.1910251 \\
IMF5 & 1.0538673 & -17.647724 & 0.5758265 & 0.1624432 \\
IMF6 & 1.0580169 & -16.825878 & 0.5473883 & 0.1371283 \\
IMF7 & 1.1282002 & -15.432964 & 0.507058 & 0.1237084 \\
IMF8 & 1.4363067 & -13.349279 & 0.3164718 & 0.116619 \\
\hline
\end{tabular}

Table 2. Sample of Entropy Calculation from Set E 


\begin{tabular}{|l|l|l|l|l|l|}
\hline \multirow{3}{*}{ Scenario } & \multirow{2}{*}{$\begin{array}{l}\text { SVM } \\
\text { Kernel }\end{array}$} & \multicolumn{4}{|c|}{ Accuracy (\%) } \\
\cline { 2 - 6 } & ShEN & REN & SEN & PE \\
\hline \multirow{3}{*}{1} & Linear & 89.4 & 95.2 & 71.2 & 87.6 \\
\cline { 2 - 6 } & Quadratic & 92.2 & 95.8 & 76.2 & 89.2 \\
\cline { 2 - 6 } & Cubic & 91.6 & 96.2 & 69.6 & 85.8 \\
\hline \multirow{3}{*}{3} & Linear & 80.3 & 96 & 70.3 & 86.7 \\
\cline { 2 - 6 } & Quadratic & 95 & $\mathbf{9 7 . 3}$ & 77.7 & 84.7 \\
\cline { 2 - 6 } & Cubic & 95.7 & 98 & 75.7 & 84.7 \\
\hline & Linear & 65 & 78 & 50.4 & 61.8 \\
\cline { 2 - 6 } & Quadratic & 70.8 & 80.2 & 52 & 61.4 \\
\cline { 2 - 6 } & Cubic & 69.2 & 79.2 & 49.2 & 59 \\
\hline
\end{tabular}

Table 3. Classification Result for Three Scenarios

\begin{tabular}{|l|c|c|c|}
\hline Scenario & Specificity & Sensitivity & Accuracy \\
\hline 1 & $99.25 \%$ & $92 \%$ & $95.8 \%$ \\
\hline 2 & $99.75 \%$ & $97 \%$ & $97.3 \%$ \\
\hline 3 & $98.25 \%$ & $92 \%$ & $80.2 \%$ \\
\hline
\end{tabular}

Table 4. REN And Quadratic SVM Performance

Each scenario was classified using the linear and non-linear kernel of SVM. The result for each scenario shown in Table 3. The table shows that the best accuracy was obtained by using REN, and quadratic SVM kernel which has $97.3 \%$ of accuracy. The detailed performance from REN and quadratic SVM kernel can be seen in Table 4

Shaikh et al., Tripathi et al. and Li et al. were generally grouping the dataset into two group, ictal and non-ictal condition. Shaikh et al. [17] able to classify non-ictal and ictal condition with $96.5 \%$ of specificity, $93.6 \%$ sensitivity, and $96.1 \%$ of accuracy. They are also able to classify normal and ictal condition with $96.7 \%$ of specificity, $95.7 \%$ of sensitivity, and $96.4 \%$ of accuracy. Tripathi et al. [18] did the same dataset grouping. They classified the ictal condition with the four other non-ictal conditions. The performance can be seen in Table 5. Li et al. [19] did the grouping into normal compared with the ictal condition, and interictal compared with the ictal condition. The performance for the first scenario shows $99.4 \%$ of specificity and $98 \%$ of sensitivity. In the second scenario, it shows $96.25 \%$ of specificity and $97 \%$ of sensitivity. Martis et al. [33] used eight IMFs as their features to classify three classes of data, normal, interictal, and ictal condition. The performance was $95.33 \%$ of accuracy.

Other entropy based research such as done by Wang et al. [41] and Kannathal et al. [42] able to classify normal, and epileptic condition with accuracy of $99.4 \%$, and $92.2 \%$ respectively. Our accuracy performance, outperfom the result from Chua et al. [43] and Wijayanto et al. [44] which able to classify three classes, normal, interictal, and ictal condition. 
Compared to the other EMD based research, our method shows a promising lead. We were able to classify more class with a fewer number of feature extraction type. The use of one type entropy (REN) in first eight IMFs of EEG signal lead to the effective use of feature numbers.

\begin{tabular}{|c|c|c|c|c|c|c|c|}
\hline Author & IMFs & $\begin{array}{l}\text { Features Extraction/ } \\
\text { No of features }\end{array}$ & Classifier & $\begin{array}{l}\text { Classified } \\
\text { Classes }\end{array}$ & SPE & SENS & ACC \\
\hline Shaikh et al. [17] & 5 & Statistic features / 15 & ANN & $\begin{array}{l}\mathrm{ABCD} / \mathrm{E} \\
\mathrm{AB} / \mathrm{E} \\
\mathrm{A} / \mathrm{E}\end{array}$ & $\begin{array}{l}96.5 \\
96.7 \\
99\end{array}$ & $\begin{array}{l}93.6 \\
95.7 \\
99.7\end{array}$ & $\begin{array}{l}96.1 \\
96.4 \\
99.3\end{array}$ \\
\hline Tripathi et al. [18] & 8 & fuzy entropy & SVM & $\begin{array}{l}\mathrm{A} / \mathrm{E} \\
\mathrm{B} / \mathrm{E} \\
\mathrm{C} / \mathrm{E}\end{array}$ & $\begin{array}{l}100 \\
100 \\
98.50\end{array}$ & $\begin{array}{l}99.75 \\
98.75 \\
98.75\end{array}$ & $\begin{array}{l}99.88 \\
99.38 \\
98.62\end{array}$ \\
\hline Li et al. [19] & 5 & $\begin{array}{l}\text { coeff of variation \& } \\
\text { fluctuation index }\end{array}$ & SVM & $\begin{array}{l}\mathrm{D} / \mathrm{E} \\
\mathrm{AB} / \mathrm{E} \\
\mathrm{CD} / \mathrm{E}\end{array}$ & $\begin{array}{l}95 \\
99.4 \\
96.25 \\
\end{array}$ & $\begin{array}{l}99 \\
98 \\
97 \\
\end{array}$ & $\begin{array}{l}97 \\
-\end{array}$ \\
\hline Martis et al. [33] & 8 & spectral features / 7 & $\begin{array}{l}\text { Decision } \\
\text { tree }\end{array}$ & $\mathrm{AB} / \mathrm{CD} / \mathrm{E}$ & - & - & 95.33 \\
\hline This work & 8 & Renyi entropy / 8 & SVM & $\begin{array}{l}\mathrm{AB} / \mathrm{CD} / \mathrm{E} \\
\mathrm{B} / \mathrm{C} / \mathrm{E} \\
\mathrm{A} / \mathrm{B} / \mathrm{C} / \mathrm{D} / \mathrm{E}\end{array}$ & $\begin{array}{l}99.25 \\
99.75 \\
98.25\end{array}$ & $\begin{array}{l}92 \\
97 \\
92\end{array}$ & $\begin{array}{l}95.8 \\
97.3 \\
80.2\end{array}$ \\
\hline
\end{tabular}

Table 5. Performance Comparison With Other Emd-based Researches

\begin{tabular}{|l|l|l|l|c|}
\hline Author & $\begin{array}{l}\text { Features / } \\
\text { no of features }\end{array}$ & $\begin{array}{l}\text { Classified } \\
\text { classes }\end{array}$ & Classifier & Acc (\%) \\
\hline Chua et al. [43] & $\begin{array}{l}\text { Bispectrum, } \\
\text { entropy / 3 }\end{array}$ & $\mathrm{AB} / \mathrm{CD} / \mathrm{E}$ & $\mathrm{SVM}$ & 93.1 \\
\hline Wang et al.[41] & $\begin{array}{l}\text { Wavelet } \\
\text { Packet } \\
\text { Entropy / }\end{array}$ & $\mathrm{AB} / \mathrm{CDE}$ & $\mathrm{GMM}$ & 92.7 \\
\hline Kannathalet al. [42] & Entropies /4 & $\mathrm{AB} / \mathrm{CDE}$ & $\mathrm{ANFIS}$ & 92.2 \\
\hline Wijayanto et al. [44] & MWPE / 5 & $\mathrm{AB} / \mathrm{CD} / \mathrm{E}$ & $\mathrm{SVM}$ & 94.3 \\
\hline Acharya et al. [45] & $\begin{array}{l}\text { Entropies, HOS, } \\
\text { Higuci FD, Hurst/7 }\end{array}$ & $\mathrm{AB} / \mathrm{CD} / \mathrm{E}$ & Fuzzy & 99.7 \\
\hline This work & Entropy / 8 & $\mathrm{AB} / \mathrm{CD} / \mathrm{E}$ & $\mathrm{SVM}$ & 95.8 \\
& & $\mathrm{~B} / \mathrm{C} / \mathrm{E}$ & & 97.3 \\
& & $\mathrm{~A} / \mathrm{B} / \mathrm{C} / \mathrm{D} / \mathrm{E}$ & & 80.2 \\
\hline
\end{tabular}

Table 6. Comparison with Other Entropy Based Researches 


\section{Conclusions}

This research describes the classification of seizure in EEG signal by using EMD and entropy as feature extraction. By using eight first IMFs from EMD process, we extract the feature by using Renyi entropy then classify the data by 5- fold crossvalidation using quadratic SVM as the classifier. The highest accuracy obtained was $95.8 \%$ of accuracy for the first scenario, $97.3 \%$ of accuracy for the second scenario, and for the third scenario, we obtained $80.2 \%$ of accuracy.

This result shows that IMFs of EEG signal has different signal complexity for normal and epileptic seizure signal. In subsequent research, we currently explore other signal complexity metric for EEG signal analysis.

\section{References}

[1] Nova, M., Vyslouzilova, L., Vojtech, Z., Stepankova, O. (2019). Towards Computer Supported Search for Semiological Features in Epilepsy Seizure Classification: World Congress on Medical Physics and Biomedical Engineering 2018, vol. 68/2. Singapore: Springer Singapore, 2019.

[2] Chang, B. S., Lowenstein, D. H. (2003). Epilepsy, N. Engl. J. Med., 349 (13), 1257-1266, September.

[3] Annegers, J. F. (2001). The Epidemiology of Epilepsy, in Wyllie E, ed. The Treatment of epilepsy: Principles and Practice, 3rd Edition, Philadelphia: Lippincott Williams \& Wilkins, 2001, 131-8.2.

[4] World Health Organization, Epilepsy: Keyfacts, 2018. [Online]. Available: http://www.who.int/news-room/fact-sheets/detail/ epilepsy. [Accessed: 13-Aug-2018].

[5] Yayasan Epilepsi Indonesia, Bunga Rampai Epilepsi di Indonesia. FKUI, 2004.

[6] Fisher, R. S. et al. (2005). Epileptic Seizures and Epilepsy: Definitions Proposed by the International League Against Epilepsy (ILAE) and the International Bureau for Epilepsy (IBE), Epilepsia, 46 (4), 470-472, April. 2005.

[7] Commission on Epidemiology and Prognosis of the International League Against Epilepsy, Guidelines for epidemiologic studies on epilepsy. Commission on Epidemiology and Prognosis, International League Against Epilepsy., Epilepsia 34 (4), $592-6$.

[8] Thurman, D. J., et al. (2011). Standards for epidemiologic studies and surveillance of epilepsy, Epilepsia, 52 (7), 2-26.

[9] Litt, B., and Echauz, J. (2002). Prediction of epileptic seizures, Lancet Neurol., 1 (1) 22-30, May.

[10] Alotaiby, T. N., Alshebeili, S. A., Alshawi, T., Ahmad, I., Abd El-Samie, F. E. (2014). EEG seizure detection and prediction algorithms: a survey, EURASIP J. Adv. Signal Process., 2014, 1, p. 183, December. 2014.

[11] Mormann, F., Andrzejak, R. G., C. E. Elger, K. Lehnertz, Seizure prediction: the long and winding road, Brain, 130 (2), 314-333, Feburuary 2007.

[12] Bou Assi, E., Nguyen, D. K., Rihana, S., Sawan, M. (2017). Towards accurate prediction of epileptic seizures: A review, Biomed. Signal Process. Control, 34, 144-157, 2017.

[13] Fahoum, F., Lopes, R., Pittau, F., Dubeau, F., Gotman, J. (2012). Widespread epileptic networks in focal epilepsies: EEG-fMRI study, Epilepsia, 53 (9), 1618-1627, 2012.

[14] Lopez, S., Suarez, G., Jungries, D., Obeid, I., Picone, J. (2015). Automated Identification of Abnormal EEGs, IEEE Signal Process. Med. Biol. Symp., 1-4, 2015.

[15] Paul, Y. (2018). Various epileptic seizure detection techniques using biomedical signals: a review, Brain Informatics, 5 (2), 6.

[16] Huang, N. E., et al. (1998). The empirical mode decomposition and the Hilbert spectrum for nonlinear and non-stationary time series analysis, Proc. R. Soc. A Math. Phys. Eng. Sci., 454 (1971), 903-995, March.

[17] Shaikh, M. H. N., Farooq, O., Chandel, G. (2019). EMD Analysis of EEG Signals for Seizure Detection, In: Lecture Notes in Electrical Engineering, 2019, 189-196.

[18] Tripathi, D., Agrawal, N. (2019). Epileptic Seizure Detection Using Empirical Mode Decomposition Based Fuzzy Entropy and Support Vector Machine, 2019, 109-118. 
[19] Li, S., Zhou, W., Yuan, Q., Geng, S., Cai, D. (2013). Feature extraction and recognition of ictal EEG using EMD and SVM, Comput. Biol. Med., 43 (7), 807-816, August 2013.

[20] Andrzejak, R. G., Lehnertz, K., Mormann, F., Rieke, C., David, P., Elger, C. E. (2001). Indications of nonlinear deterministic and finite-dimensional structures in time series of brain electrical activity: Dependence on ecording region and brain state, Phys. Rev. E - Stat. Physics, Plasmas, Fluids, Relat. Interdiscip. Top., 64 (6), 8.

[21] Rilling, G., Flandrin, P., Paulo, G. (2003).On Empirical Mode Decomposition and Its Algorithms, In: IEEE-EURASIP Workshop on Nonlinear Signal and Image Processing NSIP-03, 2003.

[22] Charleston-Villalobos, S., González-Camarena, R., Chi-Lem, G., Aljama-Corrales, T. (2007).Crackle Sounds Analysis By Empirical Mode Decomposition, IEEE Eng. Med. Biol. Mag., 26, February, 40-47.

[23] Lozano, M., Fiz, J. A., Jané. (2013). Estimation of Instantaneous Frequency from Empirical Mode Decomposition on Respiratory Sounds Analysis, In: Proceedings of the Annual International Conference of the IEEE Engineering in Medicine and Biology Society, EMBS (2013), 2013, p. 981-984.

[24] Chen, X., Shao, J., Long, Y., Que, C., Zhang, J., Fang, J. (2014). Identification of Velcro rales based on Hilbert-Huang transform, Phys. A Stat. Mech. its Appl., 401 (8), 34-44, May.

[25] Biju, K. S., Jibukumar, M. G. (2018). Ictal EEG Classification based on State Space Modeling of Intrinsic Mode functions, Procedia Comput. Sci., 125, 468-475, 2018.

[26] Gupta, V., Bhattacharyya, A., Pachori, R. B. (2017). Classification of seizure and non-seizure EEG signals based on EMDTQWT method, Int. Conf. Digit. Signal Process. DSP, 2017-August, 2017.

[27] Parvez, M. Z., Paul, M. (2014).Epileptic seizure detection by analyzing EEG signals using different transformation techniques, Neurocomputing, 145, p. 190-200, 2014.

[28] Vijayasankar, A., Kumar, P. R. (2018). Correction of blink artifacts from single channel EEG by EMD-IMF thresholding, In: 2018 Conference on Signal Processing and Communication Engineering Systems (SPACES), 2018, 2018-January, p 176-180.

[29] Shahbakhti, M., Khalili, V., Kamaee, G. (2012). Removal Of Blink From EEG By Empirical Mode Decomposition (EMD), In: The 2012 Biomedical Engineering International Conference (BMEiCON-2012), 2012.

[30] Shahbakhti, M. (2013). Evaluation of two methods based on EMD for elimination of blink from EEG, In: 2013 IEEE 33rd Int. Sci. Conf. Electron. Nanotechnology, ELNANO 2013 - Conf. Proc., p. 223-227, 2013.

[31] Zheng, D., Cui, G., Cao, J., Tanaka, T. (2015).Analysis of Brain-Death EEG Data Using 2T-EMD Algorithm, In: 2015 11th International Conference on Signal-Image Technology \& Internet-Based Systems (SITIS), 2015, 1, 528-531.

[32] Yadav, S. K., Bajaj, V., Kumar, A. (2017). An EMD based approach for discrimination of apnea and normal EEG signals, In: 2017 International Conference on Recent Innovations in Signal processing and Embedded Systems (RISE), 2017, p 191-194.

[33] MARTIS, R. J., (2012). Application Of Empirical Mode Decomposition (EMD) For Automated Detection Of Epilepsy Using EEG Signals, Int. J. Neural Syst., 22 (06), 1250027, December 2012.

[34] Sabeti, M., Katebi, S., Boostani, R. (2009). Entropy and complexity measures for EEG signal classification of schizophrenic and control participants, Artif. Intell. Med., 47, 263-274, 2009.

[35] Renyi, A. (1960). On Measures of Entropy and Information, In: Proceedings of the fourth Berkeley Symposium on Mathematics, Statistics and Probability, 1960, p 547-561.

[36] Bandt, C., Pompe, B. (2002). Permutation Entropy: A Natural Complexity Measure for Time Series, Phys. Rev. Lett., 88 (17) 174102, April.

[37] Cortes, C., Vapnik, V. (1995). Support vector machine, Mach. Learn., p. 1303-1308, 1995.

[38] Direito, B., Teixeira, C. A., Sales, F., Castelo-Branco, M., Dourado, A. (2017). A Realistic Seizure Prediction Study Based on Multiclass SVM, Int. J. Neural Syst., 27 (3), 1750006.

[39] Burges, C. J. C. (1998).A Tutorial on Support Vector Machines for Pattern Recognition, Data Min. Knowl. Discov., 2 (2), $121-167$.

[40] Nandan, M., Talathi, S. S., Myers, S., Ditto, W. L., Khargonekar, P. P., Carney, P. R. (2010).Support vector machines for seizure 
detection in an animal model of chronic epilepsy, J. Neural Eng., 7 (3), 2010.

[41] Wang, D., Miao, D., Xie, C. (2011). Best basis-based wavelet packet entropy feature extraction and hierarchical EEG classifica-ion for epileptic detection, Expert Syst. Appl., 38 (11), 14314-14320.

[42] Kannathal, N., Choo, M. L., Acharya, U. R., Sadasivan, P. K. (2005).Entropies for detection of epilepsy in EEG, Comput. Methods Programs Biomed., 80 (3), 187-194, December.

[43] Chua, K. C., Chandran, V., Acharya, U. R., Lim, C. M. (2009). Automatic identification of epileptic electroencephalography signals using higherorder spectra, Proc. Inst. Mech. Eng. Part HJ. Eng. Med., 223 (4), 485-495, April.

[44] Wijayanto, I., Rizal, A., Hadiyoso, S. (2018). Multilevel Wavelet Packet Entropy and Support Vector Machine for Epileptic EEG Classification, In: $4^{\text {th }}$ International Conference on Science and Technology, 2018.

[45] Acharya, U. R., Sree, S. V., Ang, P. C. A., Yanti, R., Suri, J. S. (2012).Application Of Non-Linear And Wavelet Based Features For The Automated Identification Of Epileptic Eeg Signals, Int. J. Neural Syst., 22 (2), 1250002, April 2012. 